\title{
Estudio Morfológico de la Retina de Salmones (Salmo salar)
}

\author{
Morphological Study of the Retina of Salmon (Salmo salar)
}

Mario Pellón*; Mariana Rojas".**; Pabla Yaikin*** Mariano del Sol*

\begin{abstract}
PELLÓN, M.; ROJAS, M. YAIKIN, P. \& DEL SOL, M. Estudio morfológico de la retina de salmones (Salmo salar). Int. J. Morphol., 33(2):788-793, 2015.
\end{abstract}

RESUMEN: La retina de peces teleósteos como pez cebra, se ha transformado en un importante modelo para el estudio de la plasticidad neuronal y la neurogénesis. Se ha demostrado además que la retina experimenta cambios ontogenéticos para adaptarse a distintos medios ambientes durante su vida. Este estudio tiene como objetivo describir el desarrollo ontogenético de la retina del alevín de salmón desde la eclosión hasta la fase de juvenil. Se trabajó con 30 salmones divididos en tres grupos de 10. Grupo I: recién eclosionados, con saco vitelino y $18 \mathrm{~mm}$ de longitud. Grupo II: sin saco vitelino y $30 \mathrm{~mm}$ de longitud. Grupo III: $100 \mathrm{~mm}$ de longitud. Cinco alevines de cada grupo fueron procesados según el protocolo de Hanken \& Wassersug para medir los diámetros dorsoventral y nasal-temporal utilizando el cartílago que protege al globo ocular. Los restantes cinco ejemplares fueron seccionados con micrótomo Microm en forma seriada $(5 \mu \mathrm{m})$ y procesados con técnica H\&E/Azul de Alcián. Se midieron las capas de la retina en un microscopio óptico Zeiss, con cámara Powershot incorporada y con un software Image Tool 3.0. El Grupo 1 presentó grandes ojos pigmentados, con aspecto de copa óptica embrionaria, la retina está estratificada en capas. La Capa Nuclear Interna (CNI) mide $62 \pm 10 \mu \mathrm{m}$ y la capa plexiforme interna (CPI) $10 \pm 2 \mu \mathrm{m}$. El Grupo 2 presenta cambios en el espesor de ellas. La CNI disminuye su espesor a $45 \pm 8 \mu \mathrm{m}$ y la Plexiforme aumenta a $25 \pm 5 \mu \mathrm{m}$. En los peces juveniles del Grupo 3, la CNI alcanza el espesor mínimo $(15 \pm 3 \mu \mathrm{m})$, por el contrario, la capa Plexiforme interna aumenta su espesor hasta alcanzar $(70 \pm 10 \mu \mathrm{m})$. En los tres grupos estudiados observamos en la periferia de la retina una zona proliferativa germinativa, que corresponde a un remanente del neuroepitelio embrionario, responsable del crecimiento continuado de la retina. La retina de los salmones puede ser también un importante modelo para el estudio de la ontogenia, la plasticidad neuronal y la neurogénesis. Esta neurogénesis en la retina de peces facilita la reordenación celular a lo largo de la ontogenia, lo que potencialmente permite la optimización del sistema visual a los cambios en las demandas visuales. Este estudio puede ser de utilidad para facilitar el diagnóstico en las patologías de ojo en salmonicultura y también puede contribuir a conocer mejor la regeneración de tejidos. Por otro lado, con estudios posteriores, la neurogénesis de la retina de peces podría extrapolarse al tratamiento de enfermedades humanas con daño a nivel retineal, tales como glaucoma, desprendimiento de retina y retinopatía diabética.

PALABRAS CLAVE: Retina; Ojo; Alevín; Peces teleósteos; Salmón; Desarrollo.

\section{INTRODUCCIÓN}

Al pasar a través de las diferentes fases del desarrollo (alevín, juvenil, adulto) los salmones van cambiando de hábitat y se van relacionando con distintos ambientes luminosos. Las etapas de alevin y juveniles se desarrollan en el agua dulce, posteriormente migran al mar y ocurre una remodelación ontogenética conocido como esmoltificación, esto implica cambios en los pigmentos visuales que pasan de ser porfirodopsina dominante a rodopsina dominante. Además, hay una pérdida de fotorreceptores ultravioleta-sensibles (Browman \& Hawryshyn, 1992; Alexander et al., 1994).

Los teleósteos tienen la capacidad regenerativa de varios tejidos entre los que se encuentra la médula espinal
(Hernández \& Rojas, 2013) y el ojo, que se regenera durante toda la vida (Carter-Dawson \& Lavail, 1979). Las nuevas neuronas, generadas por la células madre de la retina, se añaden continuamente a la retina en crecimiento (Easter \& Hitchcock, 2000). En la retina adulta de teleósteos, los progenitores de bastón se encuentran dispersos a lo largo de la retina central. No sólo son los encargados de generar bastones en una retina madura intacta, sino que ante unalesión son capaces de regenerar el tejido formando todos los tipos celulares retinianos (Otteson \& Hitchcock, 2003). En la periferia de la retina existe un grupo de células progenitoras con morfología fusiforme y con una alta tasa proliferativa (Queiroz, 2007; Sánchez, 2009).

\footnotetext{
* Programa de Doctorado en Cs. Morfológicas Universidad de La Frontera, Temuco, Chile.

** Programa de Anatomía y Biología del Desarrollo, ICBM, Facultad de Medicina, Universidad de Chile, Santiago, Chile.

Este estudio fue parcialmente financiado a través del Proyecto DIUFRO 14-0042, Universidad de La Frontera, Chile.
} 
Dentro de la retina, los pigmentos visuales absorben la luz e inician una cascada de fototransducción que conduce a la señalización neuronal y en última instancia el comportamiento del pez. Los pigmentos visuales son moléculas sensibles a la luz que se encuentran en los segmentos externos de los fotorreceptores y consisten en un cromófobo basado en la vitamina A unido a una proteína opsina. Se han identificado cuatro clases de opsinas de cono en los vertebrados: sensible a ultravioleta (SWS1), sensible a longitud de onda corta (SWS2), similares a rodopsina de longitud de onda media $(\mathrm{Rh} 2)$ y sensibles a longitud de onda larga (LWS) (Yokoyama, 2000).

La retina de peces teleósteos como pez cebra, se ha utilizado para el estudio de la plasticidad neuronal y la neurogénesis. No existen muchos estudios en retina de alevín de salmón (especie de gran importancia económica para el país). Debido a esto los objetivos de este estudio son describir el desarrollo ontogenético de la retina de este pez (posteclosional, alevín sin saco y juvenil) con el fin de establecer las características morfológicas de la retina central y la zona proliferativa periférica.

\section{MATERIAL Y METODO}

Se trabajó con 30 salmones divididos en tres grupos. Grupo I: 10 alevines recién eclosionados de $18 \mathrm{~mm}$. Grupo II: 10 alevines de $30 \mathrm{~mm}$ de longitud. Grupo III: 10 alevines de $100 \mathrm{~mm}$ de longitud. Todo el procedimiento fue realizado según las normas bioéticas de la Facultad de Medicina, Universidad de La Frontera, para la experimentación con animales de laboratorio. Los salmones fueron eutanasiados por exposición a Benzocaína al 5\%, diluida en agua (Kalmagin 20®, Farquímica). Los alevines fueron fotografiados y medidos. Cinco alevines de cada grupo fueron procesados según el protocolo de diafanización propuesto por Hanken \& Wassersug (1981) para la tinción de cartílago y hueso. Los ejemplares diafanizados fueron observados en una lupa estereoscópica (Stemi DV4, Zeiss) para evaluar el ojo y medir los diámetros dorsoventral y nasal-temporal, luego fotografiados en formato digital con cámara adaptada a la lupa (Powershot Megapixeles). Los restantes cinco ejemplares fueron incluidos en parafina para realizar cortes seriados coronales y sagitales de $5 \mu \mathrm{m}$ de grosor que luego fueron montados en portaobjetos para su tinción. De cada espécimen, se tomó uno de cada diez cortes hasta completar un total de 10 cortes por portaobjeto, los que fueron procesados mediante técnicas histológicas (H\&E/Azul de Alcián. Las imágenes fueron obtenidas con microscopio Zeiss y cámara digital Powershot. Las mediciones de las capas de la retina fueron realizadas en la retina central mediante el uso del software Image Tool 3.0 (http://compdent.uthscsa.edu/dig/ itdesc.html), pero la descripción se realizó tanto en retina central como periférica.

\section{RESULTADOS}

Los alevines del Grupo 1, presentan ojos con forma de copa óptica embrionaria, incluso destaca una fisura coroidea (Fig. 1A). Los ojos presentan gran tamaño (1,5 x $1,0 \mathrm{~mm})$ en relación a la cabeza $(3,0 \times 3,0 \mathrm{~mm})$. Se puede evidenciar un mayor espesor en la zona ventral nasal $(0,6$ $\mathrm{mm})$, en comparación con la dorsal $(0,2 \mathrm{~mm})$. En la Figura 1B se observa que el mayor espesor de la zona ventral nasal corresponde a la zona periférica germinativa de la retina periférica. La retina central presenta capa pigmentaria, capas nucleares y plexiformes, la capa ganglionar se identifica a partir de la fisura coroidea. La capa nuclear interna (CNI) mide $62 \pm 10 \mu \mathrm{m}$ y la capa plexiforme interna (CPI) $10 \pm 2$ $\mu \mathrm{m}$ (Ver Tabla I).

En el Grupo 2, el ojo mide 2,0 x 1,5 mm en comparación con la cabeza que mide 4 x $4 \mathrm{~mm}$. Con técnica de diafanización se identifica que el bulbo ocular queda completamente protegido por una órbita cartilaginosa (Fig. 1C). La retina está estratificada en capas celulares y plexiformes. Se puede identificar la capa pigmentaria de la retina y la retina neurosensorial (Fig. 1D). El epitelio pigmentario y la capa de los segmentos de los fotorreceptores tienen pigmentos de melanina (Fig. $2 \mathrm{~A} \mathrm{y}$

Tabla I. Comparación del espesor de las capas de la retina $(\mu \mathrm{m})$ según el desarrollo de alevín de salmón (X土DS).

\begin{tabular}{lccc}
\hline Capa de la Retina & Grupo 1 & Grupo 2 & Grupo 3 \\
\hline CP & $4 \pm 1$ & $16 \pm 4$ & $5 \pm 3$ \\
CNE & $5 \pm 0,6$ & $16 \pm 4$ & $10 \pm 2$ \\
CPE & $3 \pm 0,5$ & $6 \pm 2$ & $10 \pm 0,3$ \\
CNI & $62 \pm 10$ & $45 \pm 8$ & $15 \pm 3$ \\
CPI & $10 \pm 2$ & $25 \pm 5$ & $70 \pm 10$ \\
CG & $15 \pm 2$ & $19 \pm 2$ & $15 \pm 6$ \\
\hline
\end{tabular}




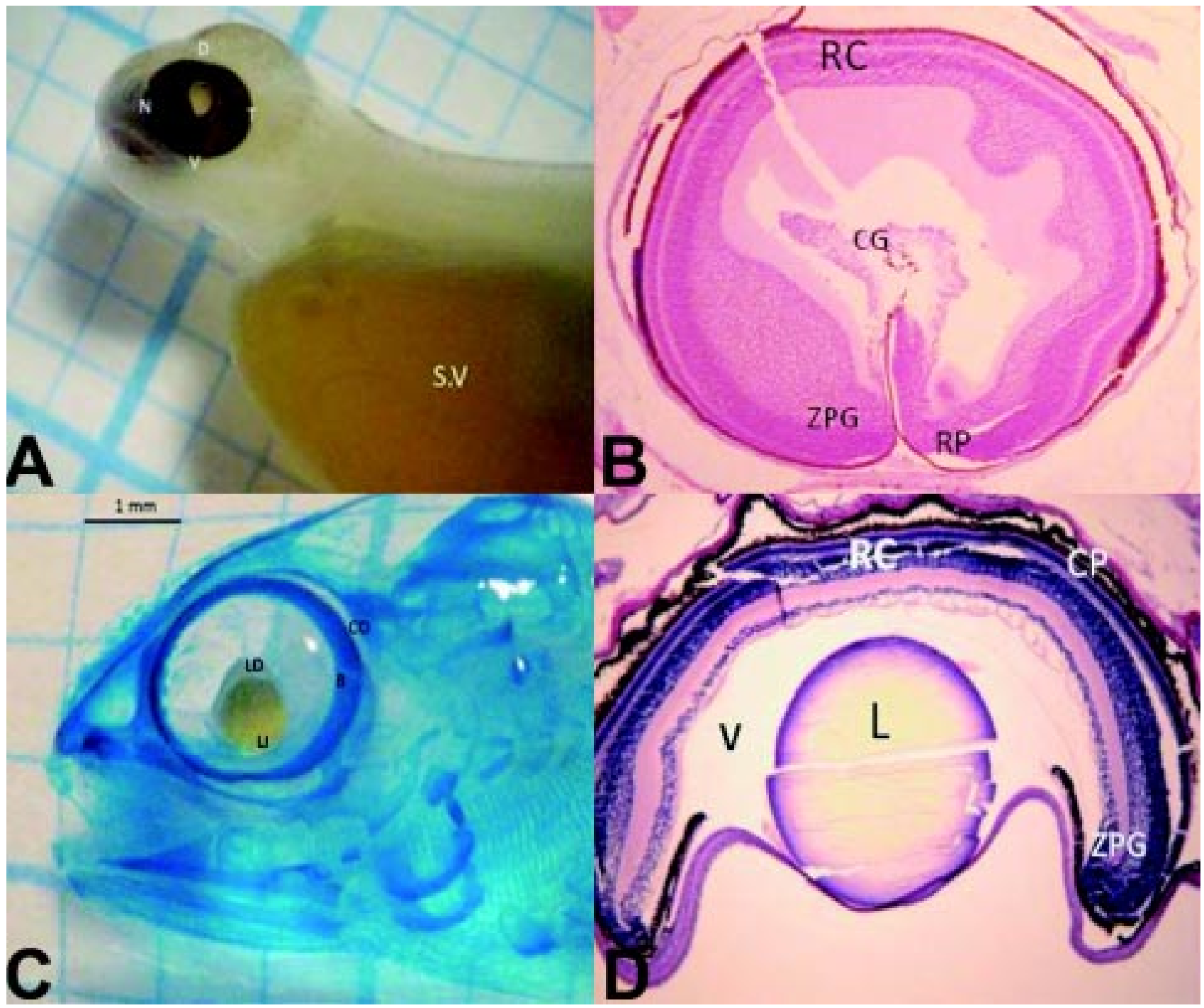

Fig. 1. A). Alevín de salmón Grupo 1. Ejes del ojo $\mathrm{N}=$ nasal, $\mathrm{T}=$ temporal, $\mathrm{D}=$ dorsal, $\mathrm{V}=$ ventral. $\mathrm{SV}=$ saco vitelino. $\mathrm{B})$. Retina del grupo $1, \mathrm{RP}=$ retina periférica, $\mathrm{ZPG}=$ zona proliferativa germinativa, $\mathrm{RC}=$ retina central, $\mathrm{CG}=$ capa ganglionar. $\mathrm{C}$ ). Alevín $\mathrm{Grupo} 2 . \mathrm{CO}=$ cavidad orbitaria, $\mathrm{B}=$ bulbo, $\mathrm{LD}=$ lente derecho, $\mathrm{LI}=$ lente izquierdo. Técnica Haenckel y Wassersug. D). Retina de alevín de Grupo 2. Se observan ZPG: zona proliferativa germinativa, RC: retina central, CP: capa pigmentaria, V vítreo, L: lente H-E-azul de Alcián 50X

B) y forman la capa pigmentaria. La membrana limitante externa es imperceptible. La capa nuclear externa está constituida por los cuerpos celulares de conos principalmente. La Capa plexiforme externa es imperceptible en alevines, es donde se producen las conexiones sinápticas entre los fotorreceptores y los diferentes tipos de interneuronas. En la capa nuclear interna se localizan los somas de todas las interneuronas retinianas, tiene el mayor espesor, presentando dos zonas una muy densa y otra más laxa (Figs. 2A y B). La Capa plexiforme interna y contiene los axones de las células bipolares, así como las dendritas de las células amacrinas, interplexiformes, ganglionares y células de microglía (Figs. 2A y B). La Capa de las células ganglionares está compuesta por los somas de las células ganglionares y células amacrinas desplazadas. La Capa de las fibras del nervio óptico constituida por los axones de las células ganglionares y células gliales. Por último se localiza la membrana limitante interna que es imperceptible (Ver Tabla I para observar espesor de las capas).

En las retinas del Grupo 3 (juvenil) el ojo mide 8,0 x $6 \mathrm{~mm}$, se observa sólo el epitelio pigmentario con melanina, a diferencia de la capa de los fotorreceptores que ya no está pigmentada. La capa nuclear interna ha reducido su espesor alcanzando el espesor mínimo (15 3 $\mu \mathrm{m})$. La capa plexiforme interna continúa siendo la de 


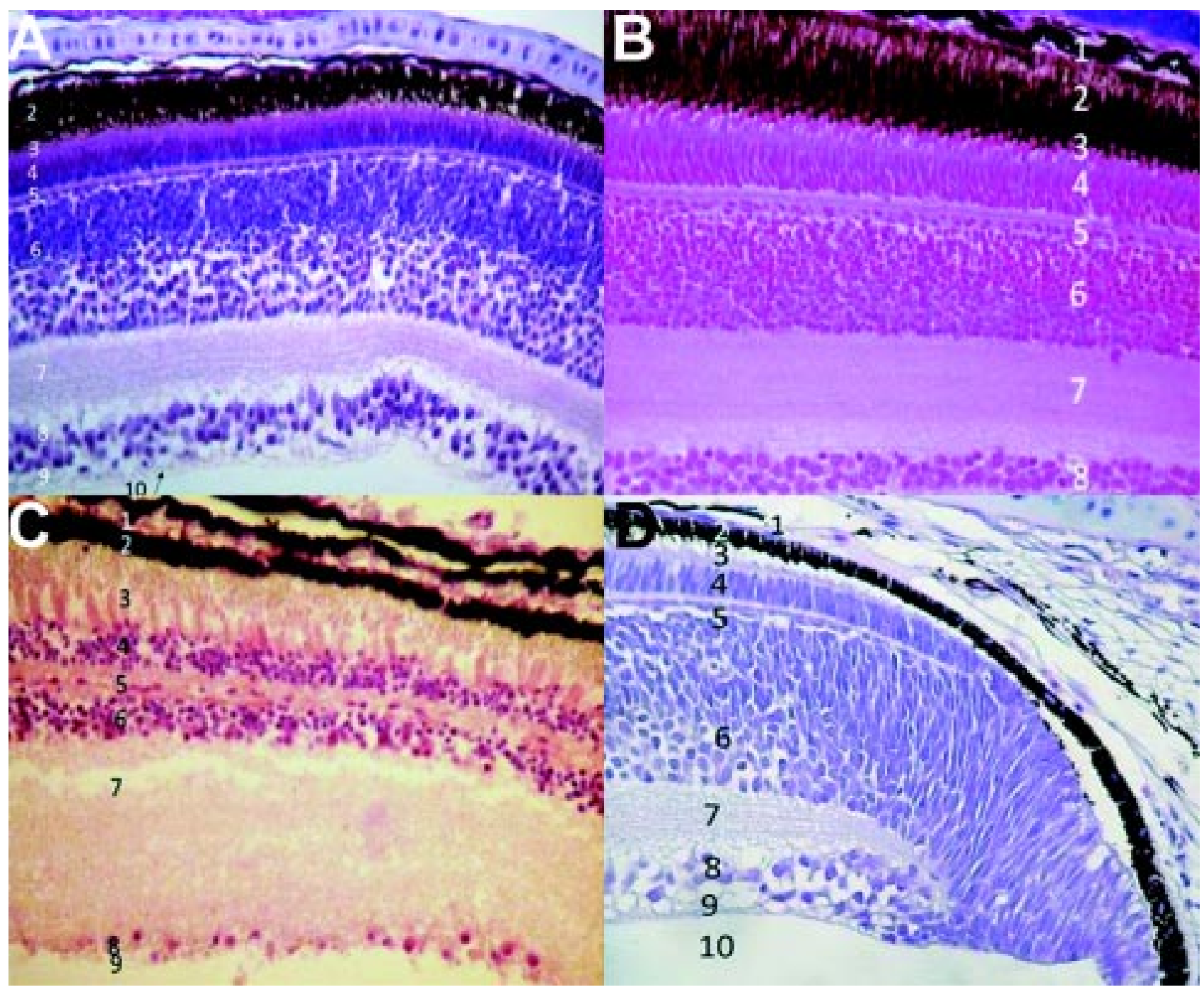

Fig. 2. A y B). Retina central de alevín de Grupo 2. C). Retina central de alevín de Grupo 3. D). Retina periférica de alevín Grupo 2. $1=$ epitelio pigmentario, $2=$ capa de los segmentos de los fotorreceptores, $3=$ membrana limitante externa. $4=$ capa nuclear externa, $5=$ capa plexiforme externa, $6=$ capa nuclear interna, $7=$ capa plexiforme interna, $8=$ capa de las células ganglionares, $9=$ capa de las fibras del nervio óptico, $10=$ membrana limitante interna. H-E, 400X.

mayor espesor $(70 \pm 10 \mu \mathrm{m})$, prácticamente la mitad de la retina (Fig. 1C) (Ver Tabla I).

En los tres grupos, se encontraron diferencias entre la zona central de la retina y la retina periférica (Figs. 1D y 2D) es así como la membrana limitante externa, la capa nuclear externa y la capa plexiforme externa que estaban presentes en la zona central no se observan en la zona periférica germinativa. La zona periférica germinal se ve como un neuroepitelio pseudoestratificado. Se encontró una zona de transición donde la laminación no está aún establecida y las células son fusiformes y se están diferenciando. Existe una clara continuidad entre la zona periférica germinal y la capa ganglionar (Fig. 2D).

\section{DISCUSION}

El Grupo 1 corresponde a alevines recién eclosionados, de grandes ojos pigmentados. Los alevines en esta etapa se mantienen inmóviles al fondo del agua (Hernández \& Rojas) y se nutren gracias al saco vitelino, al igual que otras especies (Miranda et al., 2014). La retina se encuentra estratificada en capas, pero algunas de ellas son muy difíciles de observar (plexiforme externa). Esto es muy diferente a lo que ocurre en la lubina (Queiroz) que presenta al eclosionar una retina muy indiferenciada y sin organización en capas. Solo al tercer día aparecen los gránulos de melanina, sin diferenciación de receptores. La dorada 
(Carassius auratus) recién eclosionada posee una retina neural laminada pero los fotorreceptores están poco diferenciados (Johns, 1982). Otro teleósteos como el trompetista de Tasmania (Latris lineata) presenta una retina totalmente indiferenciada al eclosionar pero a partir del tercer día aparece una clara estratificación (Coberoft \& Pankhurst, 2003). Según Queiroz todas las especies tienen sus retinas organizadas en capas celulares, pero el tiempo de formación depende de cada especie. El aspecto de copa óptica con características embrionarias solo se ha reportado en los salmónidos y se reconoce una fisura coroidea, una zona de retina central y otra periférica.

En el Grupo 2, el saco vitelino se ha reducido y el alevín puede nadar en la superficie, presenta cambios en el espesor de las capas de la retina. En comparación con el Grupo 1, disminuye el espesor de la CNI, pero aumenta a nivel de CPI. En ambos grupos, la capa de mayor grosor corresponde a la CNI, pero la CPI crece más en el Grupo 2, correspondiendo a la segunda capa más gruesa, a diferencia del Grupo 1, donde dicho lugar es ocupado por la CG (Tabla I).

Los cambios observados en las capas de la retina del Grupo 3, son similares a los reportados para la lubina por Queiroz, donde las dos capas plexiformes tienden a aumentar e incluso en el caso de este estudio, la CPI pasa a ser la capa de mayor espesor.

En la periferia de la retina observamos características similares en los tres grupos, células con morfología fusiforme, constituyendo la zona proliferativa germinativa, que corresponde a un remanente del neuro epitelio embrionario. Esto es similar a lo descrito en la retina adulta donde Stenkamp (2011) describe que se generan nuevas células a partir de dos poblaciones de retinoblastos, la zona periférica germinal y el linaje de los progenitores de bastones. Nuestros estudios también fueron similares a los reportados por Queiroz y Sánchez que indican que desde la ZPG, se van añadiendo en la retina, anillos concéntricos de células, que maduran y se diferencian, aumentando el tamaño de la retina. De esta forma, las células que se encuentren en las regiones más periféricas de la retina serán las más jóvenes. Las nuevas capas van desplazando a las ya formadas, habiendo así una zona de transición donde la laminación no está aún establecida y las células se están diferenciando. Desde la ZPG se forman todos los tipos celulares retinianos excepto los bastones (Ottenson \& Hitchcock).

La retina de los salmones puede ser también un importante modelo para el estudio de la plasticidad neuronal y la neurogénesis. Esta neurogénesis en la retina de peces facilita la reordenación celular a lo largo de la ontogenia, lo que potencialmente permite la optimización del sistema visual a los cambios en las demandas visuales. Este estudio puede ser de utilidad para facilitar el diagnóstico en las patologías de ojo en salmonicultura y también puede contribuir a conocer mejor cómo transcurre la regeneración de tejidos. Por otro lado, con estudios posteriores, la neurogénesis de la retina de peces podría extrapolarse al tratamiento de enfermedades del ojo humano, tales como glaucoma, desprendimiento de retina y retinopatía diabética, entre otras.

PELLÓN, M.; ROJAS, M.; YAIKIN, P. \& DEL SOL, M. Morphological study of the retina of salmon (Salmo salar). Int. J. Morphol., 33(2):788-793, 2015.

SUMMARY: The retina of teleost fish zebrafish, has become an important model for studying neuronal plasticity and neurogenesis. It was further shown that the retina undergoes ontogenetic changes to adapt to different environments during their lifetime. This study aims to describe the ontogenetic development of the retina of juvenile salmon from hatching to the juvenile stage. We worked with 30 salmon divided into three groups of 10 . Group I: newly hatched with yolk sac and $18 \mathrm{~mm}$ in length. Group II: without yolk sac and $30 \mathrm{~mm}$ in length. Group III: $100 \mathrm{~mm}$ long. Five fry each group were processed according to the protocol of Hanken \& Wassersug to measure dorsoventral and nasal-temporal diameters using the cartilage that protects the eyeball. The remaining five specimens were sectioned with a microtome Microm serially $(5 \mu \mathrm{m})$ and processed with technical H-E / Alcian blue. The layers of the retina were measured on a Zeiss optical microscope with camera Powershot built and with Image Tool 3.0 software. Group 1 showed large pigmented eyes, looking embryonic optic cup, the retina is stratified in layers. The inner nuclear layer $(\mathrm{CNI})$ measured $62 \pm 10$ microns and the inner plexiform layer (CPI) $10 \pm 2 \mu \mathrm{m}$. Group 2 presents changes in the thickness of them. The CNI decreases in thickness to $45 \pm 8 \mu \mathrm{m}$ and the plexiform increased to $25 \pm 5 \mu \mathrm{m}$. In juvenile fish of group 3, the CNI reaches the minimum thickness $(15 \pm 3 \mu \mathrm{m})$, by contrast, the inner plexiform layer thickness increases up to $(70 \pm 10 \mu \mathrm{m})$. In the three groups observed in the periphery of the retina one proliferative germinative zone, which corresponds to a remnant of the embryonic neural epithelium responsible for the continued growth of the retina. The retina of the salmon can also be an important model for the study of ontogeny, neuronal plasticity and neurogenesis. This retinal neurogenesis fish rearrangement facilitates cell along ontogeny, potentially allowing optimization of the visual system to changes in the visual demands. This study may be useful to help diagnose pathologies in eye salmon and can also contribute to better understand tissue regeneration. On the other hand, with later studies, fish's retinal neurogenesis could be extrapolated to the treatment of human retinal diseases, such us glaucoma, retinal detachment o diabetic retinopathy.

KEY WORDS: Retina; Eye; Teleost Fish; Salmon; Development. 


\section{REFERENCES}

Alexander, G.; Sweeting, R \& Mckeown, B. The shift in visual pigment dominance in the retinae of juvenile coho salmon (Oncorhynchus kisutch): an indicator of smolt status. J. Exp. Biol., 195(1):18597, 1994.

Browman, H. I. \& Hawryshyn, C. W. Thyroxine induces a precocial loss of ultraviolet photosensitivity in rainbow trout (Oncorhynchus mykiss, Teleostei). Vision Res., 32(12):2303-12, 1992.

Carter-Dawson, L. D. \& LaVail, M. M. Rods and cones in the mouse retina. II. Autoradiographic analysis of cell generation using tritiated thymidine. J. Comp. Neurol., 188(2):263-72, 1979.

Cobcroft, J. M. \& Pankhurst, P. M. Sensory organ development in cultured striped trumpeter larvae Latris lineata: implications for feeding behaviour. Mar. Freshw. Res., 54(5):669-82, 2003.

Easter, S. S. Jr. \& Hitchcock, P. F. Stem cells and regeneration in the retina: What fish have taught us about neurogenesis. Neurosci., 6(6):454-64, 2000.

Hanken, J. \& Wassersug, R. The visible skeleton. A new double-stain technique reveals the native of the "hard" tissues. Funct. Photogr., 16:22-6, 1981.

Hernández, H. \& Rojas, M. Development of spinal cord of the salmon (Salmo salar) during the period post eclosional. Int. J. Morphol., 31(1):172-6, 2013.

Johns, P. R. Formation of photoreceptors in larval and adult goldfish. J. Neurosci., 2(2):178-98, 1982.

Miranda, L.; Vilaxa, A.; Ávila, R. \& Rodríguez, M. Characterization of the stages in the cycle of embryo development Sarda chiliensis chiliensis (Alva, 1987) in an aquatic re-circulation system (SAR). Int. J. Morphol., 32(4):1492-501, 2014.

Otteson, D. C. \& Hitchcock, P. F. Stem cells in the teleost retina: persistent neurogenesis and injury-induced regeneration. Vision Res., 43(8):927-36, 2003.

Queiroz, G. R. G. Morfogénesis y plasticidad sináptica retiniana de larvas de lubina (Dicentrarchus labrax) adaptadas a la luz y a la oscuridad. Tesis Doctoral. Alicante, Departamento de Biotecnología, Universidad de Alicante, 2007.

Sánchez, G. M. R. Caracterización in vivo e in vitro de progenitores neuronales en la retina de peces teleósteos. Tesis Doctoral. Salamanca, Tesis del Departamento de Biología Celular y Patología, Universidad de Salamanca, 2009.

Stenkamp, D. L. The rod photoreceptor lineage of teleost fish. Prog. Retin. Eye Res., 30(6):395-404, 2011.

Yokoyama, S. Molecular evolution of vertebrate visual pigments. Prog. Retin. Eye Res., 19(4):385-419, 2000.

\author{
Dirección para Correspondencia: \\ Mario Pellón Arcaya \\ Facultad de Medicina \\ Universidad de La Frontera \\ Casilla 54-D. Temuco \\ CHILE
}

Email: mario.pellon@ufrontera.cl

Recibido : 22-01-2015

Aceptado: 14-03-2015 